\title{
Health and nutritional status of adolescent girls: A study of beneficiaries of rural areas of Bihar in India
}

\author{
Kusum Bharti, Manoj Kumar and Pramila Prasad
}

\begin{abstract}
Adolescent girls are the worst sufferers of the ravages of various forms of malnutrition because of their increased nutritional needs and low social power. Scheme of Adolescent Girls, is an important scheme of the Ministry of Women and Child Development, Govt. of India for the improvement of health and nutritional status of Adolescent Girls under the platform of ICDS. Objective: This study aimed at assessing nutritional anaemia of Adolescent girls of rural areas of Banka district receiving the benefits of the nutritional intervention of SABLA/SAG yojna under the ICDS and other related schemes. Method: For this study, Anthropometry measurement, 24h dietary recall method including Take Home Rashan of SABLA programme, Haemoglobin Level, SABLA Kishori Card Observation and Questionnaire method as tools were used. Result :Out of 500 AGLs from SABLA of 50 Rural AWCs, 79 per cent are underweight whereas 67 per cent stunted. The nutritional status of 85 per cent is undernourished i.e. according to the BMI only 15 per cent girls are in normal range. 78 per cent AGLs are anaemic in which 24 per cent moderate and 9 per cent are severe anaemic i.e. Hb level $<7 \mathrm{~g} / \mathrm{dl}$. When the dietary availability of AGLs was assessed, we found that only 32 per cent AGLs are getting adequate diet according to the RDA, whereas 62 per cent girls are getting THR according to the norms of SABLA scheme. The General appearance of only 28 per cent AGLs is normal, whereas 68 per cent are thin and 4 per cent sickly. Only 32 per cent AGLs maintain the personal hygiene and sanitation. More than 80 per cent AGLs under SABLA at Banka district are undernourished whereas 85 per cent are anaemic. It may be concluded that this scheme at studied area is not being implemented in a proper way.
\end{abstract}

Key Words : Dietary intake, Nutrition, Adolescent girls, Anaemia, Undernutrition

How to cite this article : Bharti, Kusum, Kumar, Manoj and Prasad, Pramila (2020). Health and nutritional status of adolescent girls: A study of beneficiaries of rural areas of Bihar in India. Food Sci. Res. J., 11(2): 103-107, DOI : 10.15740/HAS/FSRJ/11.2/103107.Copyright@2020: Hind Agri-Horticultural Society. 